\title{
The Effectiveness of an On-campus Open House Targeting Underrepresented Students
}

\section{Dr. La'Tonia Stiner-Jones, Ohio State University}

Dr. Stiner-Jones is Assistant Dean of Graduate Programs and Assistant Professor of Practice in Biomedical Engineering at The Ohio State University's College of Engineering. As Assistant Dean she provides leadership of graduate affairs and professional development for graduate students and postdoctoral trainees. She also oversees strategic recruitment of graduate students with a focus on increasing diversity. As Assistant Professor, she is responsible for participating in teaching, scholarship and service for the department.

Dr. Stiner-Jones received her Bachelor's and PhD. degrees from Wright State University and her MBA from Capital University. After completing her PhD in Biomedical Sciences, she completed postdocs, in neuroimmunology and psychoneuroimmunology at Ohio State. Her work has been published in numerous scientific journals and presented both nationally and internationally. After completing her postdoctoral fellowship, Dr. Stiner-Jones accepted a faculty position in Ohio State's College of Dentistry and served as Director of Minority Student Recruitment and DENTPATH, a post baccalaureate program to prepare disadvantaged students for dental school. 


\title{
The Effectiveness of an On-Campus Open House Targeting
}

\section{Underrepresented Students}

\begin{abstract}
The study highlights the outcomes of a program to increase diversity of the application pool by bringing prospective students to campus early in the application cycle. We identified active and early engagement of female and underrepresented minority prospective students in an on-campus event as a way to interest them in our graduate programs. During the one- to two-day event students have the opportunity to engage with faculty, staff and students in their program(s) of interest, prepare for the application process and learn about funding and faculty research. The demographics of the event have grown from $22 \%$ to $35 \%$ female and $39 \%$ to $44 \%$ underrepresented minority. Participants were provided post-event surveys to assess their understanding of the graduate admissions process and graduate school. In fact, $100 \%$ of respondents who participated in the workshop on preparing an admissions application, indicated they had a better understanding of how to prepare one after attending the workshop. Of respondents who participated in the event, over $90 \%$ indicated the event influenced there decision to apply and nearly 90\% indicated their intent to apply. Furthermore, 96.9\% indicated they would recommend the event to a friend. We have been hosting this event for 4 years and between 50-65\% of female and underrepresented minority attendees ultimately apply and are admitted. We are continuously seeking ways to improve the event based on participant, faculty, staff and graduate student feedback.
\end{abstract}

\section{Introduction}

According to the National Science Foundation, women and underrepresented minorities (African Americans, Hispanics, and Native Americans) represent $24.1 \%$ and $6.4 \%$ of the engineering graduate student population nationally [1]. These data confirm the need to increase the participation of these groups in engineering graduate education. With representation of underrepresented minority students and women so low, there are concerns about the ability to create the most innovative solutions to the most challenging engineering questions in society. However, increasing representation of underrepresented groups in engineering is a multi-faceted issue that extends beyond getting students into the application pool, it extends to admission and graduation. Therefore, the solution to the issue of underrepresentation is likely to be multifaceted as well and approaches to address these issues are currently being investigated. Here we address the issue of low representation of these students in our applicant pool by developing an open house. Research indicates, that many minority students lack knowledge about the admissions process to doctoral programs, have an interest in building social support networks in graduate school and view a commitment of financial support and the involvement of faculty in recruitment as important [2]. This event addresses these key issues and serves to connect students to our programs early. In doing so, we bring prospective students to campus during the application season to help them prepare to submit their applications to graduate school and interact with our faculty, staff and students. The objective of the open house is to recruit participants to apply to our engineering graduate programs. Though we actively recruit juniors, 
senior undergraduates represent the majority of our program attendees so that we may recruit them immediately into our graduate programs. This study examined the following 1 ) whether bringing diverse students to our campus prior to them applying to graduate school would increase the number of underrepresented students who apply and are admitted to our programs and 2) the impact of the event on all participants' preparation to apply regardless of demographic group. Our results indicate the event has increased the numbers of underrepresented minority and female students who apply and are admitted to our graduate programs. Moreover, attendees regardless of demographic group had a better understanding of the application process and funding as a result of participating.

\section{Open House Program}

Female, underrepresented minority and majority domestic junior and senior students from across the country were invited to apply to attend the open house. To recruit students we contacted them directly using our email lists comprised of lists we purchased, acquired through name exchanges and prior attendees of our Research Experience for Undergraduates program. We sent invites to colleagues at target institutions and the advisors of the Society of Women Engineers, National Society of Black Engineers, and Society of Hispanic Professional Engineers at target institutions for dissemination. We also sent the invitation to the Women in Engineering ProActive Network and the National Association of Multicultural Engineering Program Advocates list serves for dissemination by program coordinators and directors. Originally, the program was one day long. The event began with a welcome and breakfast. Afterward, students attended a poster session and workshops about how to prepare their application and funding opportunities. Next, attendees met with current graduate students followed by departmental visits.

Two years ago, we redesigned the program and extended it to two days. We did so to increase engagement of attendees and our programs. These changes are mentioned solely to show how the two programs differed. The application and admission rates of attendees of both formats are consistently high. The redesigned event retained all the existing content except the poster session. Instead of poster sessions we incorporated faculty led research sessions. The changes allowed prospective students to have more interactions with our programs, faculty and graduate students and visit our engineering centers. During the first day, we hosted conference style workshops with multiple opportunities for students to learn about research and talk to faculty one on one or in group settings. We also added underrepresented minority and female graduate student panel discussions, so prospects from these groups could learn about being a graduate in our college from the perspective of students in these demographic groups. On day two, students tour the engineering centers of their choosing, which offered an opportunity to see research applications, their impact and learn the role graduate students play at engineering centers.

\section{Methods}

We assessed program effectiveness using post-survey, graduate application and admission, and program attendance data. An assessment of program effectiveness was conducted following the one- and two-day open house events. This study examined the following 1) whether bringing female and underrepresented minority students to our campus early in the application cycle would increase the numbers of these students who apply to our programs and 2) the impact of the 
event on participant preparation to apply, regardless of demographic group.

Event attendance, graduate program application and admission, and Qualtrics online survey data were collected and analyzed. Figure 1 illustrates the post-survey questions. The post-survey was disseminated within one week of event completion. The Institutional Review Board submission for this study is in preparation.

Figure 1.

Post-Survey Questions

1. Did the "Crafting a Competitive Application" workshop give you a better understanding of how to create a competitive application?

a. Yes

b. No

2. Did the "How to Fund Your Education" workshop give you a better understanding of funding options for graduate students?

a. Yes

b. No

3. Do you intend to apply to OSU College of Engineering to pursue a graduate degree?
a. Yes
b. Maybe
c. No

4. How likely are you to recommend this event to a friend?
a. Extremely likely
b. Somewhat likely
c. Neutral
d. Somewhat unlikely
e. Extremely unlikely

\section{Results}

The data indicate that this program increases participant understanding of the application process and funding and the number of student's from underrepresented groups who apply and are admitted to our graduate programs. One hundred percent of respondents indicated the workshop on completing the application improved their understanding and $96 \%$ of respondents indicated improved understanding of funding after attending the corresponding workshop (Figure 2). After attending the event, 94\% of respondents indicated the event influenced their decision to apply and nearly $90 \%$ indicated their intent to apply (Figure 3). When asked whether they would recommend the event to a friend, $96.9 \%$ of students indicated they would (Figure 4). Though we have not assessed whether past participants actually did recommend the event, in the future we plan to ask former participants to forward our invite to a friend. 

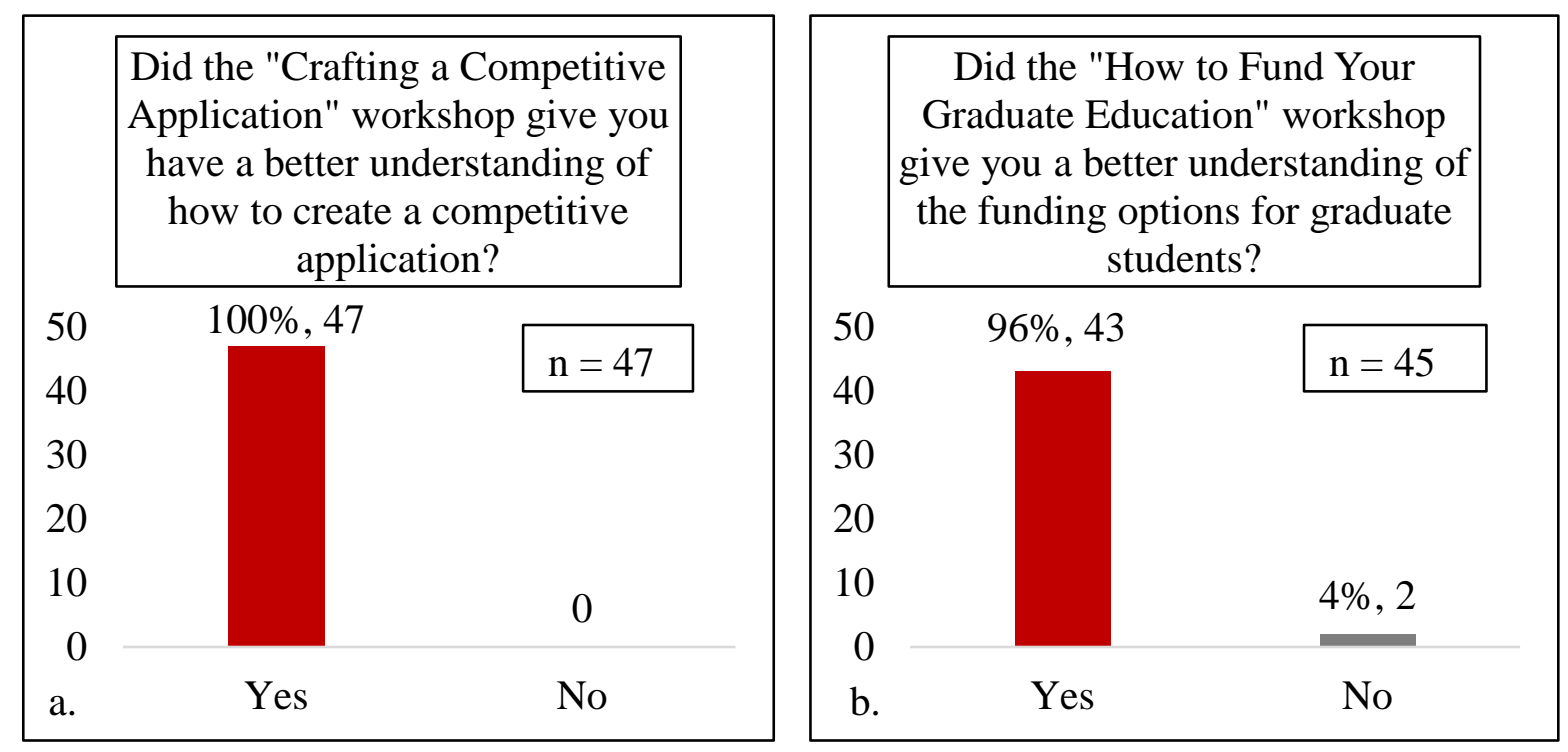

Figure 2. Illustrates the effectiveness of the open house workshops.

The demographics of the event have grown from $22 \%$ to $35 \%$ female and $39 \%$ to $44 \%$ underrepresented minority (Figure 5). On average between $50-65 \%$ of female and underrepresented minority attendees apply and are admitted. Female and underrepresented minority open house participants increased graduate applications to our college by $3 \%$ and $15 \%$, respectively.

Admission to graduate engineering programs is highly competitive. The rate of admission for the entire population of students who attend our open houses ranges from $60 \%-68 \%$

compared to $16.6 \%-18.3 \%$ for all applicants who applied to our college during the same period. Taken together these data indicate the effectiveness of our program at getting students to apply and that students who participate are more likely to be admitted to one of our programs. We also attempted to determine where attendees who did not apply to our college ended up. The response rate to this survey was less than $20 \%$ but of those who responded $40 \%$ applied to graduate school somewhere else and 60\% joined the workforce. 


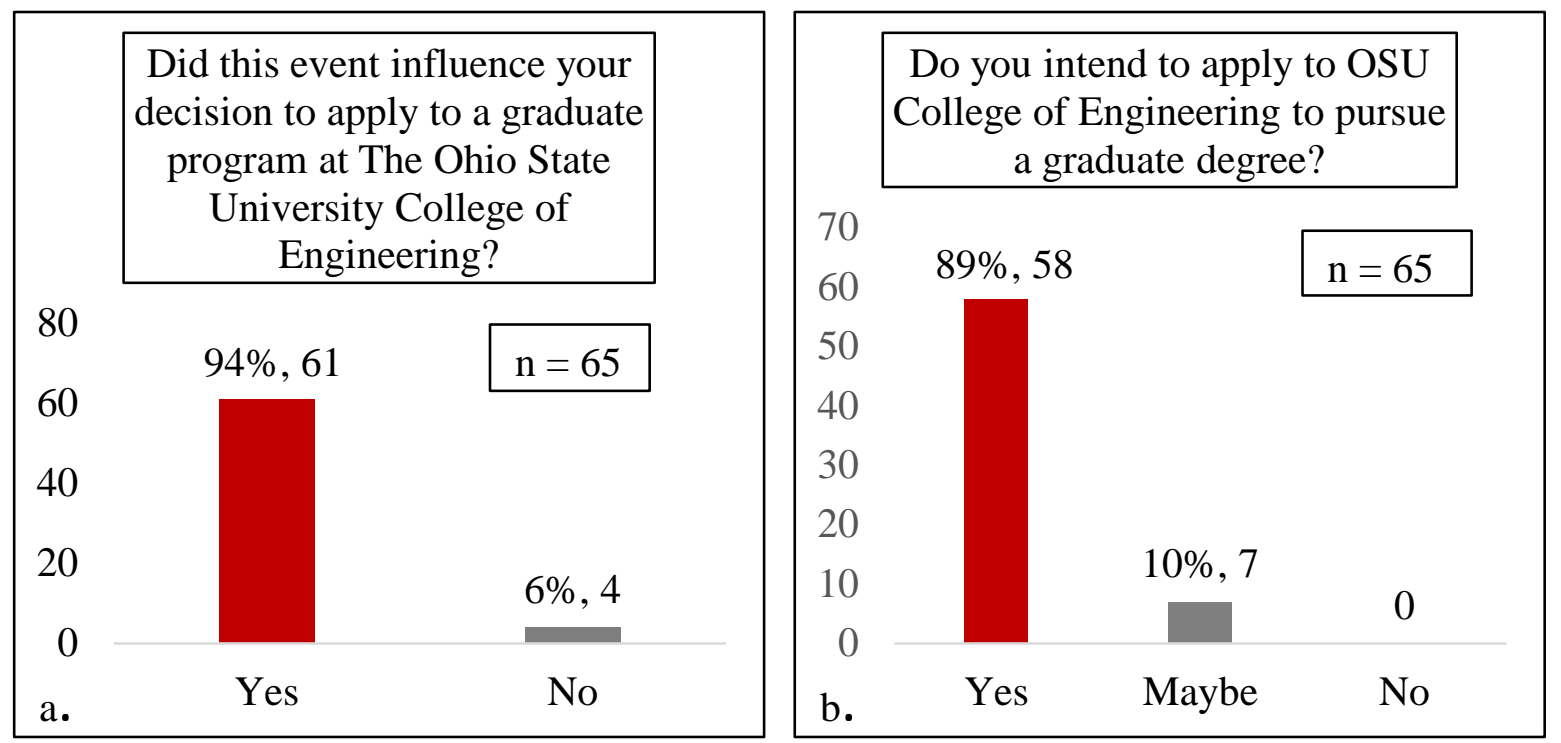

Figure 3. Illustrates whether the event influenced participant's decision to apply and whether participant's intended to apply.

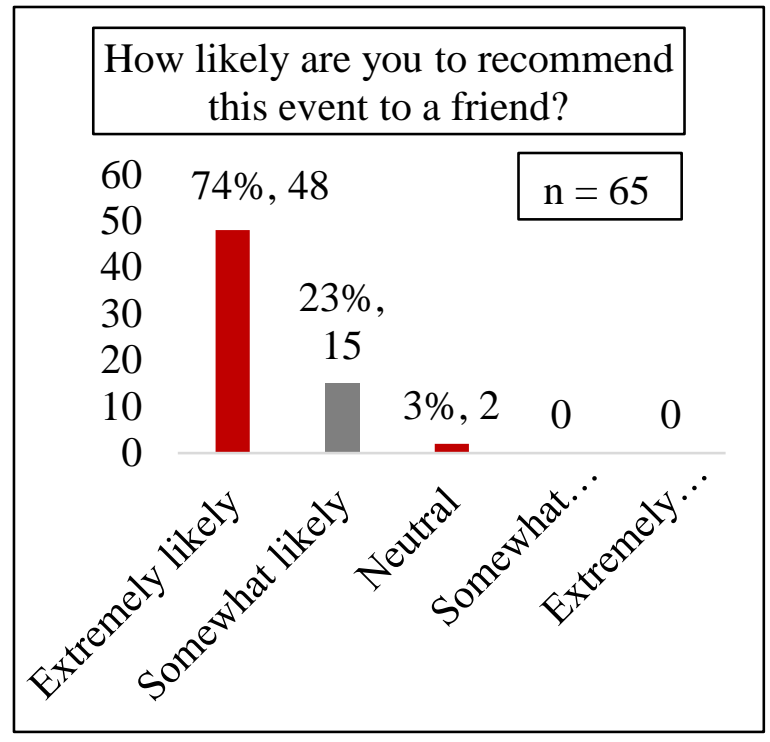

Figure 4. Illustrates the likelihood of participants to recommend the event. 
2013 Open House by Gender

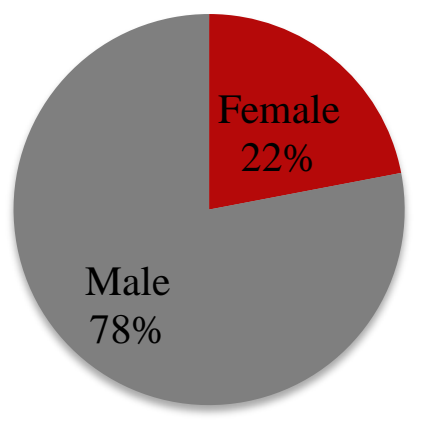

2013 Open House by Race/Ethnicity

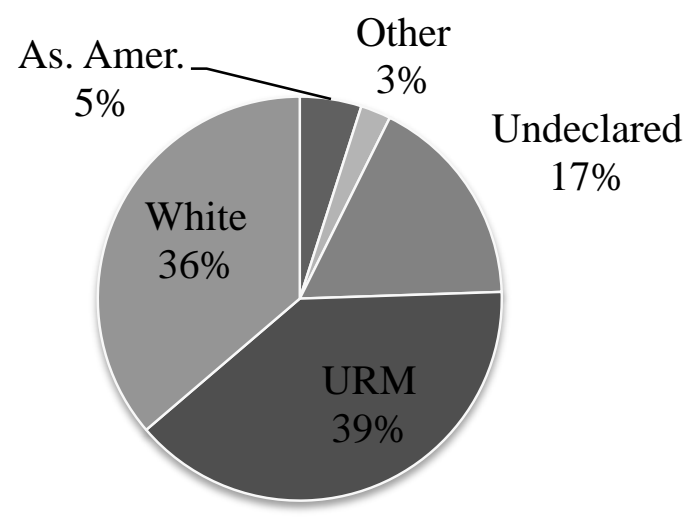

2017 Open House by Gender

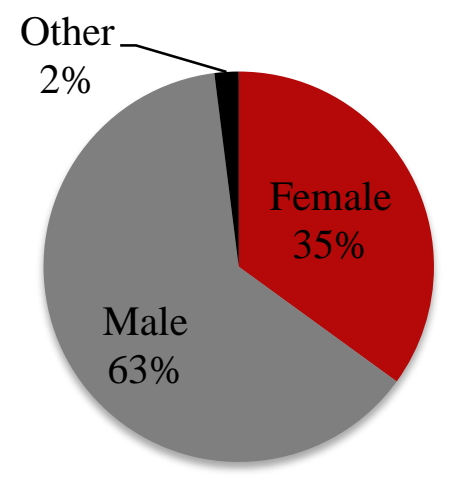

2017 Open House by Race/Ethnicity

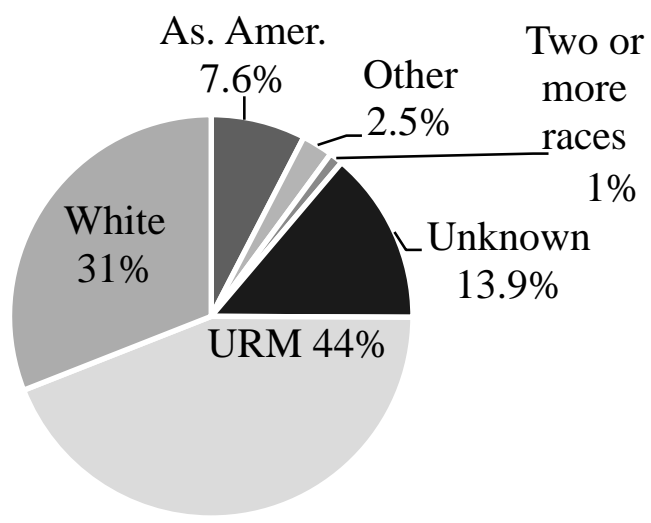

Figure 5. Illustrates the change in demographics for the open house events over time. (As. Amer. = Asian American and URM = underrepresented minority)

\section{Conclusion}

The representation of women and underrepresented minorities in graduate engineering programs nationally is well below their representation in society. Achieving greater participation requires creative new strategies to proactively engage these students early in the application cycle. The open house serves as an effective model to address this issue. Though, we have made great strides in enhancing our diversity there is still more to be done. 


\section{Future Work}

This is a work in progress. Moving forward we aim to expand the magnitude and impact of the event by allowing more students to attend the program and will investigate differences in program impact by demographic group. We will also investigate the impact our event had on students preparation to apply to other institutions. 


\section{References}

[1] National Science Foundation, National Center for Science and Engineering Statistics, "Women, Minorities, and Persons with Disabilities in Science and Engineering”, Special Report NSF 17-310. Arlington, VA. Available at www.nsf.gov/statistics/wmpd/. 2017.

[2] K. Farmbry, "Expanding the Pipeline: Explorations on Diversifying the Professoriate", Journal of Public Affairs Education, vol. 13, no.1, pp. 115-132, Winter 2007. 